\title{
A Comprehensive Study and Performance Analysis of Facsimile Transmission using GSM Fixed Wireless Terminal
}

\author{
Manju Jose \\ Research Scholar \\ Mother Teresa Women's University \\ India
}

\begin{abstract}
Facsimile continues to be communication bedrock for transmitting sensitive business critical documents with legal standing. Traditional facsimile uses PSTN network for communication. As the mobile technology advances, fixed wireless terminals are being used to connect landline devices such as telephone, fax machines etc and transmit using the mobile phone networks. Fixed wireless terminals are used in locations where traditional landline infrastructure is unavailable. This paper presents the performance analysis of facsimile transmission using GSM fixed wireless terminal under varying conditions to determine the margins of the network parameters that enable successful communication. The metrics Facsimile Satisfactory Rate parameter was used for the evaluation of the quality of facsimile transmission using GSM fixed wireless terminal. The analysis was carried out under varying traffic conditions to study the effect of congestion on the facsimile transmission using GSM fixed wireless terminal, at varying distances from the base station both in the rural and urban area. The results showed that the facsimile transmission using GSM fixed wireless terminal is dependent on the terrain, the distance of the fixed wireless terminal from the base station and the traffic volume.
\end{abstract}

\section{General Terms}

Wireless Communication

\section{Keywords}

Facsimile transmission; GSM fixed wireless terminal; Fax; Facsimile Satisfactory Rate; SS7; G3 facsimile.

\section{INTRODUCTION}

Facsimile transmission persists to be reliable technology for business communication. The facsimile device scans the original document, converting it into a bitmap and then transmits it as electrical signals through the telephone system (PSTN). The receiving facsimile device converts the coded image back to its normal form and produces a hard copy. Group 3 facsimile also known as G3 facsimile is the more commonly used format now [1]. G3 facsimile supports one dimensional image compression of black and white images. In FoIP (Fax over Internet Protocol) the fax is transmitted through internet rather than the traditional method of sending faxes through the telephone line. A common method of faxing now is the computer based faxing where the document is send as email attachment [2]. Facsimile transmission using GSM fixed wireless terminal uses the GSM network. It is especially useful where there is no wired infrastructure like marine communication, remote office, oil related field, military use etc. The popularity of GSM has rapidly increased the facsimile transmission using GSM fixed wireless terminal. Facsimile devices were optimized for communication over

\author{
S. K. Srivatsa \\ Department of Electronics \& communication \\ St. Joseph's College \\ India
}

PSTN. There have been some efforts to evaluate Fax over IP (FoIP) performance in a packet based network. Nevertheless, there have been little efforts to investigate the performance of facsimile transmission using GSM fixed wireless terminal. The facsimile device is connected to the GSM fixed wireless terminal as shown in Figure1. The fixed wireless terminal acts as a GSM gateway and routes the fax over the mobile network. Later the fax is switched to the PSTN network and thereby it reaches the destination.

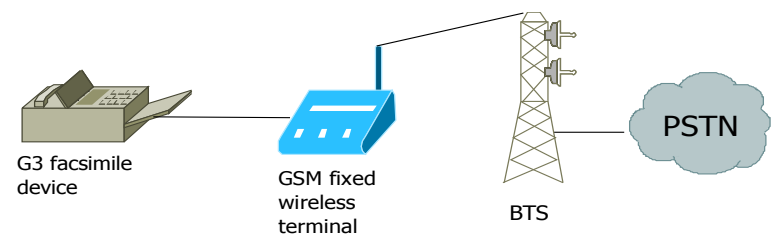

Figure1. Scenario for facsimile transmission using GSM fixed wireless terminal

Performance measurements provide authentic data for network planning, monitoring and maintenance. The KPIs used in GSM service are paging success rate, TCH (traffic channel) congestion rate, handover success rate, call setup delay etc. All these KPI are influenced by the traffic volume in the network. Congestion is a daily focus of network optimization and is a key factor affecting the GSM performance [3].

Only limited work has been reported in the field of performance evaluation of facsimile transmission. [4] reported the performance evaluation of facsimile in a packet based network. The research work [5] provides a basic framework internet fax transmission. The paper [6] has studied the utilization of SIP (Session Initiation Protocol) in conjunction with SDP (Session Description Protocol) as companion protocol to SIP to exchange real-time fax. [7] studied the use of T.38 facsimile in conjunction with SIP for transmission of fax traffic over IP networks. [8] proposed an architecture and development efforts in constructing a real-time implementation of multicasting in fax over Internet.

Radio propagation is a term used to explain how radio waves behave when they are transmitted, or are propagated from one point to another. Radio propagation is heavily site specific and can vary significantly depending on the terrain [9]. Radio waves are affected by the phenomena of reflection, refraction, diffraction, absorption and scattering. Radio propagation in open areas is much different from the radio propagation in indoor and urban areas. In urban areas the shortest direct path (the line of sight [LOS]) between the transmitter and receiver is usually blocked by buildings and other terrain features 
outdoors. Similarly in indoor areas walls, floors and interior objects within buildings obstruct LOS communications. Such scenarios are called non-LOS (NLOS) or obstructed LOS (OLOS). This further complicates radio propagation in these areas, and the signal is usually carried by a multiplicity of indirect paths with various signal strengths. The signal strength of these paths depends on the distance they have traveled, the obstacles they have reflected from or passed through, the architecture of the environment and the location of objects around the transmitter and receiver. This study focuses on the performance analysis on facsimile transmission using GSM fixed wireless terminal under varying traffic conditions and at varying distances from the base station in the rural and urban area.

This paper is organized as follows: The procedure for the facsimile transmission using GSM fixed wireless terminal is given in Section 2. Test setup and methodology is given in Section 3. Section 4 represents the results and discussion of facsimile transmission using GSM fixed wireless terminal under varying traffic conditions at various distance from the base station both in rural and urban area. Section 5 includes the summary and conclusion.

\section{TECHNIQUE FOR FACSIMILE TRANSMISSION USING GSM FIXED WIRELESS TERMINAL}

GSM employs cellular concept. In a cellular concept the coverage area is separated into a group of contiguous smaller areas. Each of these smaller areas has its own base stations. These areas have radio channels assigned in a clever way to reduce the interference. These smaller areas are called cells. Groups of cells are called clusters. Every cluster uses the completely offered radio spectrum. A number of low-power base stations are used for transmission, each of which has a limited coverage area. When a new base station or transmitter is set up, the available capacity is multiplied because the same spectrum is reused. Theoretically a cell radius can be upto $35 \mathrm{~km}$, but practically that is not achievable.

To describe such a wireless network system a reference model or overall architecture is required. This delivers a complete understanding of the network elements and operation. This also distributes the system into subsystems [9]. The following are the three subsystems as shown in Figure 2:

- Mobile Station (MS): Mobile Station is used for communicating with the user. It amends the information from the user to the transmission protocols of the airinterface, so that it can communicate with the BSS. Subscriber Identity Module (SIM) and Mobile Equipment (ME) are the two components of MS. SIM is a smart card which identifies the specifications of a user such as address and type of service. SIM is issued at the subscription time. $\mathrm{ME}$ is a hardware that can be purchased from the manufacturer.

- Base Station Subsystem (BSS): The BSS connects the wired infrastructure and the user through the wireless airinterface. This is accomplished using the wired protocols. The Base Transceiver Station (BTS) and the Base Station Controller (BSC) are the two components of BSS. BTS is located in the midpoint of the cells where the BSS antenna is installed. The BTS has a transmitter, a receiver, and signaling equipment which assist it to function over the airinterface. The Base Transceiver Station works along with the MS for physical communication over the air-interface.
Base Station Controller is a small switch inside the BSS. BSC is responsible for frequency administration and handover among the BTSs inside a BSS.

- Network and Switching Subsystem (NSS): The NSS is accountable for network operation. It helps for registration and maintenance of the connection with the MSs. It also offers for communications with other wired and wireless networks. The NSS consists of one hardware Mobile Switching Centre (MSC), and four software elements such as Visitor Location Register (VLR), Home Location Register (HLR), Equipment Identification Register (EIR), and Authentication Center (AUC). A MSC is the hardware part of the wireless switch. This switch communicates with PSTN switches using the signaling system-7 (SS-7) protocol. It also communicates with other MSCs in the coverage area of a service provider. HLR is the database software. This software holds the management of the mobile subscriber account. This stores the subscriber's address, service type, current location forwarding address, authentication/ciphering keys, and billing information. VLR is the temporary database software similar to the HLR. It is used for recognizing the subscribers visiting inside the coverage area of an MSC. The AUC handles different algorithms that are used for authentication and encryption of the subscribers. The EIR is another database that is used for managing the identification of the mobile equipment against faults and theft. EIR holds the international mobile equipment identity (IMEI) that discloses the manufacturer, country of production, and terminal type. These information can be used to report stolen phones. This can also be used to check if the phone is operating according to the specification of its type[9].

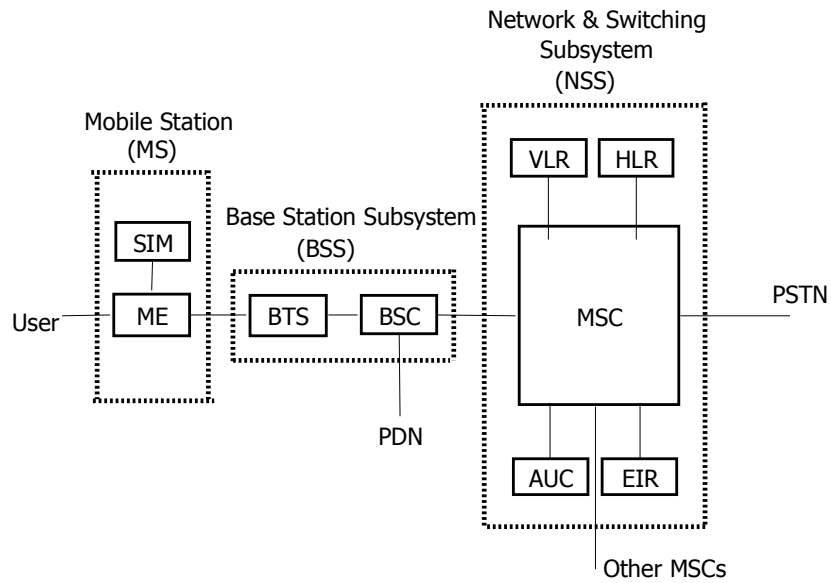

Figure2. Reference architecture of GSM

Fax service is the transmission of facsimile (image) information between users. Facsimile signals are normally sent using audio frequencies which cannot be sent through the GSM voice coder. To send fax signals through the GSM system, the GSM system must decode the fax signal and send it in digital form. When the fax data reaches its GSM exit point, the GSM system must reconvert the digital fax back into it original analog form.

The facsimile devices are connected to the GSM fixed wireless terminal using a phone cord with RJ 11 connector. The facsimile communication service needs to be registered with the respective network operator. The facsimile number for the SIM card is different from the voice telephone number. Users can send/receive faxes through this fax machine and the operating is same as the ordinary fax machine. The key to 
delivering the facsimile services through GSM is something called SS7 (Signaling System Number 7) [10]. SS7 is a set of protocols which provides fast, efficient, reliable transfer and delivery of signaling information across the GSM network. It supports both switched voice and non-voice applications [11]. SS7 provides extremely fast data connections among mobile switching centres, so fast that the networks can obtain enhanced services data while the call is being connected. GSM network supports fax speeds of up to $9,600 \mathrm{bps}$. The data and fax components of the networks support all the international data and fax standards like Group 3 fax, v.32 and v. 27 ter. V.32 is a modem which operates as full duplex on a 4 wire circuit or half duplex on a 2 wire circuit. It is an ITU-T recommendation and allows bidirectional data transfer at 9600 bit/s or $4800 \mathrm{bit} / \mathrm{s}$ at a symbol rate of $2400 \mathrm{baud} \mathrm{[12].} \mathrm{V.27} \mathrm{is}$ also an ITU-T recommendation but for half duplex modem and allows only 2400 and $4800 \mathrm{bit} / \mathrm{s}$ [13].

\section{PERFORMANCE ANALYSIS OF FACSIMILE TRANSMISSION USING GSM FIXED WIRELESS TERMINAL}

A detailed analysis of facsimile transmission using GSM fixed wireless terminal was carried out based on the following parameters: varying distances from the base station and varying traffic conditions. This analysis was performed both in rural and urban areas because the radio propagation vary significantly depending on the terrain and the site. The test environment was created using the regular facsimile devices and the public GSM network. It also used a regular GSM fixed wireless terminal and thus made a real-life environment for the testing purpose.

\subsection{Test Setting}

The test setting as shown in Figure3 was constructed to evaluate the performance under varying parameters. The test setting used the public GSM network service provided by the best network operator throughout the experiment. The PSTN network used for the experiment was also from the same provider. GSM frequency band gives the radio spectrum frequencies assigned by the ITU for the operation of GSM. GSM 900 frequency band was used for the experiment as provided by the network operator [14]. The uplink frequency band is $890-915 \mathrm{MHz}$ and the downlink is $935-960 \mathrm{MHz}$. Group 3 facsimile device (Samsung SF-650) which is popular in the market was used for the study. The GSM fixed wireless terminal (FT22) was used throughout the study.

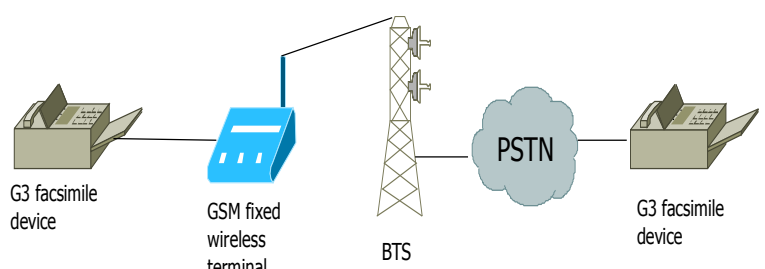

Figure3. Test setting

The experiments were conducted at times when the required parameters are met and that it was confirmed from the service provider. The study was conducted by the transmission of facsimile images from a Group 3 facsimile device through the GSM fixed wireless terminal via the GSM network to PSTN and then the destination Facsimile device under various network parameters such as traffic, distance from the base station and terrain. The study was conducted to evaluate the efficiency of facsimile transmission using GSM fixed wireless terminal by analyzing various parameters of image transmission, like image quality, transmission speed, transmission success rate under varying network conditions. The metrics Facsimile Satisfactory Rate (FSR) was used for the evaluation [4].

\subsection{Test Methodology}

The test image used for the experiment is as shown in Figure4 was chosen as per [4]. It is a standardized, digitized image set with high density and patterns that offer enough observation points for the analysis of image quality as given in [15]. The quality of a transmitted image $\left(\mathrm{F}_{\mathrm{IQ}}\right)$ evaluation was done as demonstrated [4]. Ten observation points were defined. These 10 observation points were assigned 2 points if all the details of the observation point were clear, 1 point if some were clear and some were blurred, and 0 if most of the observation points were not clear.

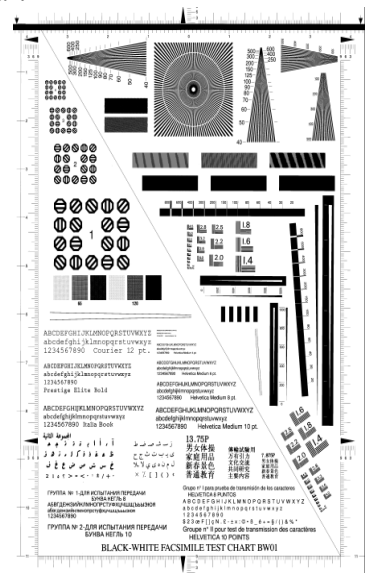

Figure4. The testing image 9 from ITU-T T.24.

\subsection{Test Image and Overall Efficiency Evaluation}

As demonstrated [4], normalized transmission time and transmission success rate were also used to analyze the efficiency of facsimile transmission using GSM fixed wireless terminal. Average transmission time $\left(\mathrm{F}_{\mathrm{TT}}\right)$ is the difference between the start time of the facsimile transmission $\left(t_{\text {start }}\right)$ and the end time of the successful transmission at the receiving facsimile device $\left(t_{\text {end }}\right)$ :

$F_{T I}=t_{\text {end }}-t_{\text {start }}$

The values $t_{\text {end }}$ and $t_{\text {start }}$ were obtained from the facsimile transmission report on the fax device. The Normalized Transmission Time $\left(\mathrm{F}_{\mathrm{NTT}}\right)$ is the ratio of $\mathrm{F}_{\mathrm{TT}}$ and $\mathrm{F}_{\mathrm{TTO}}$ parameters:

$F_{N T T}=\frac{F_{T T}}{F_{T T O}}$

where $\mathrm{F}_{\text {Tто }}$ was defined as the average measured transmission time in the case of ideal network condition, when there is no congestion and the site is close to the BTS.

The $F_{S R}$ parameter was defined as the ratio of number of successful transmission $\left(\mathrm{n}_{\mathrm{ok}}\right)$ to the total number of facsimile documents sent $\left(\mathrm{n}_{\mathrm{fax}}\right)$ :

$F_{S R}=\frac{n_{o k}}{n_{f a x}}$

Facsimile Satisfactory Rate parameter (FSR) is given by: 
$F S R=\frac{F_{S R} \times F_{I Q}}{F_{\text {NTT }}}$

Eighty subjects, who are familiar with the facsimile communication, were interviewed to conclude whether they agree with the values 16.88 points for $\mathrm{F}_{\mathrm{IQ}}, 0.8$ for $\mathrm{F}_{\mathrm{SR}}$ and 1.1 for $F_{\text {NTT }}$ as given in [4]. As all the interviewees agreed to the above value for each parameter, the acceptable value of FSR is 12.27 .

Congestion in GSM network is the unattainability of network for a subscriber to subscribe the service. There are different types of congestion in GSM network [16,17].Traffic channel congestion (TCH congestion) is caused when there is no free traffic channel. The traffic channel carries speech and data traffic. Other factors that could lead to congestion are old hardware, inadequate radio channels and infrastructure, redialing of subscribers when they face blocking, too many subscribers on the network etc. In order to study the effect of congestion the experiment was conducted when the traffic volume was $15 \%, 60 \%$ and $85 \%$ of the channel capacity. The cell radius used for the experiment was $7 \mathrm{~km}$. In order to study the effect of distance of the terminal from the base station, the experiment was conducted at various distances from the base station such as $1 \mathrm{~km}, 2 \mathrm{~km}, 3 \mathrm{~km}, 4 \mathrm{~km}, 5 \mathrm{~km}, 6 \mathrm{~km}$ and $7 \mathrm{~km}$. To analyze the effect of terrain in the GSM propagation, the experiments were conducted both in the rural and urban area using the above said parameters. The experiments were done when the traffic is $15 \%$ of the actual capacity are indicated as cap $/ 15 \%$ and that at $60 \%$ are indicated as cap $/ 60 \%$ and that at $85 \%$ are indicated as cap $/ 85 \%$.

\section{RESULTS \& DISCUSSION}

The experimental results are organized in the following two sections such as rural area and urban area.

\subsection{Rural Area}

As given in Figure 5 the $\mathrm{F}_{\mathrm{IQ}}$ parameter measurements showed that the image quality are within the acceptable range (18.7 to 18 ) when there was $15 \%$ of channel capacity utilization within the cell radius of $7 \mathrm{~km}$. The image quality was within the acceptable range (18.7 to 17.8 ) when there was $60 \%$ of channel capacity utilization within the cell radius of $7 \mathrm{~km}$. Also the image quality was within the acceptable range (18.7 to 17.5 ) when there was $85 \%$ of channel capacity utilization within the cell radius of $7 \mathrm{~km}$. There was no much degradation in the quality of the image (fonts, lines, circles, figures etc). The $\mathrm{F}_{\mathrm{NTT}}$ parameter was constant for a distance of $3 \mathrm{~km}$ when there was $15 \%$ of channel capacity utilization and it increased up to $7 \mathrm{~km}$. Still it was within the acceptable range (1 to 1.09). The $\mathrm{F}_{\text {NTT }}$ parameter was constant for a distance of $2 \mathrm{~km}$ when there was $60 \%$ of channel capacity utilization and it increased constantly up to $7 \mathrm{~km}$. Still it was within the acceptable range (1 to 1.1). The $\mathrm{F}_{\mathrm{NTT}}$ parameter was constant for a distance of
$2 \mathrm{~km}$ when there was $85 \%$ of channel capacity utilization and then there was significant increase. It was within the acceptable range until $4 \mathrm{~km}$ ( 1 to 1.09 ). From $5 \mathrm{~km}$ to $7 \mathrm{~km}$ it was above the acceptable range $(1.2$ to 1.6$)$. The $F_{S R}$ parameter was always above 0.8 which revealed that the increase in traffic volume did not cause significant number of unsuccessful transmissions.

The analysis of FSR parameter revealed that $15 \%$ utilization and $60 \%$ utilization of the channel resources did not affect the fax transmission. But when the traffic was high (85\% capacity utilization) the transmission was not affected upto $6 \mathrm{~km}$. At 7 $\mathrm{km}$ there was a major degradation in the transmitted fax. This was due to high value of $F_{\text {NTT }}$ parameter at $7 \mathrm{~km}$. This discloses that the increase in traffic (congestion) definitely affects the quality of the facsimile transmission which is the same as in the case of GSM voice transmission. In all the cases the FSR value decreased when the distance of the wireless terminal from the base station increased.

\subsection{Urban Area}

As in the case of rural area, the $\mathrm{F}_{\mathrm{IQ}}$ parameter measurements showed that the image quality are within the acceptable range (18.8 to 17.7) when there was $15 \%$ of channel capacity utilization within the cell radius of $7 \mathrm{~km}$ which is given in Figure6. In this case the range of $\mathrm{F}_{\mathrm{IQ}}$ values was higher than that of rural area. The same was the case when there was $60 \%$ of channel utilization in the urban area. The $\mathrm{F}_{\mathrm{IQ}}$ parameter was within the acceptable range (18.7 to 17.1$)$ but with a wider range of values. Unlike the previous cases the $\mathrm{F}_{\mathrm{IQ}}$ parameter was not within the acceptable range when there was $85 \%$ of capacity utilization. The $\mathrm{F}_{\mathrm{IQ}}$ parameter was within the acceptable range until a distance of $6 \mathrm{~km}$. But the image quality degraded $\left(\mathrm{F}_{\mathrm{IQ}}=16.7\right.$ which is less than acceptable value 16.88 ) when the distance was $7 \mathrm{~km}$. The $\mathrm{F}_{\text {NTT }}$ parameter was within the tolerable range when there was $15 \%$ capacity utilization. But this was not the case when the traffic volume increased ( $60 \%$ and $85 \%$ capacity utilization). In the former the $\mathrm{F}_{\mathrm{NTT}}$ parameter was within the tolerable range ( 1 to 1.1$)$ for a distance of $5 \mathrm{~km}$ and it increased further significantly. In the latter case $\mathrm{F}_{\text {NTT }}$ parameter was within the tolerable range (1.07 to 1.1) for a distance of $4 \mathrm{~km}$ and it increased further significantly. The $\mathrm{F}_{\mathrm{SR}}$ parameter was within the acceptable range in the various traffic conditions even though it declined when the distance of the fixed wireless terminal from the base station increased.

The study of FSR showed that in the urban area its value was within the tolerable range only when the traffic volume was very low (15\%). When the traffic volume was $60 \%$ and $85 \%$ there was degradation in the facsimile quality due to significant increase in $\mathrm{F}_{\mathrm{NTT}}$. When there was $60 \%$ traffic the FSR value was within the tolerable range (18.7 to 12.45) up to $6 \mathrm{~km}$. But when the traffic was $85 \%$ the FSR value was within the tolerable range (17.48 to 13.13 ) only until a distance of $5 \mathrm{~km}$ from the base station and there was significant degradation further. From this experiment it was clear that the urban area suffered more degradation in quality during the radio propagation for facsimile transmission. 

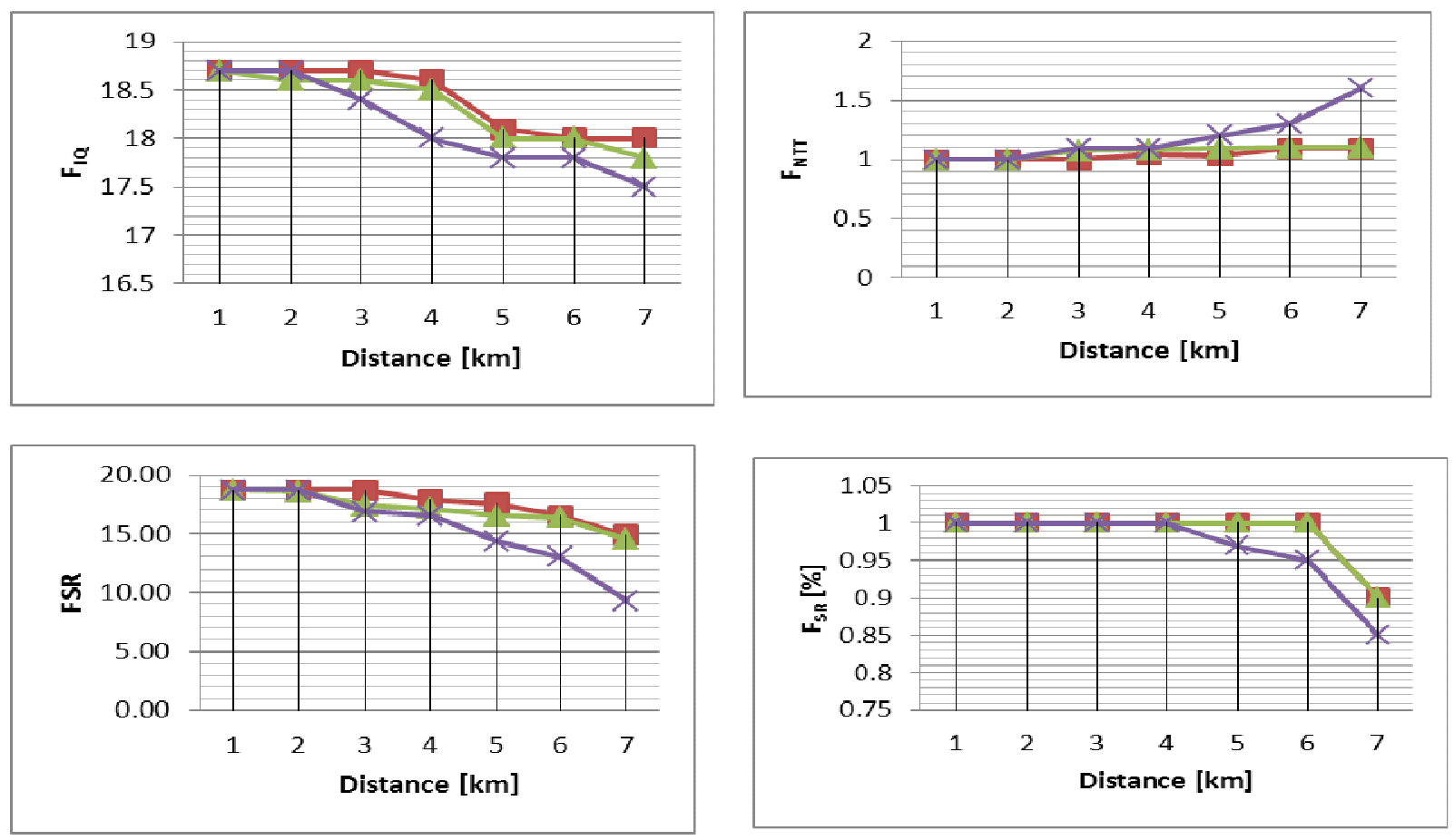

$\mathrm{Cap} / 15 \% \multimap \mathrm{Cap} / 60 \% \multimap \mathrm{Cap} / 85 \%$

Figure5. Influence of distance and traffic volume on the $F_{\mathrm{IQ}}, \mathrm{F}_{\mathrm{NTT}}, \mathrm{F}_{\mathrm{SR}}$ and FSR parameters in rural area.
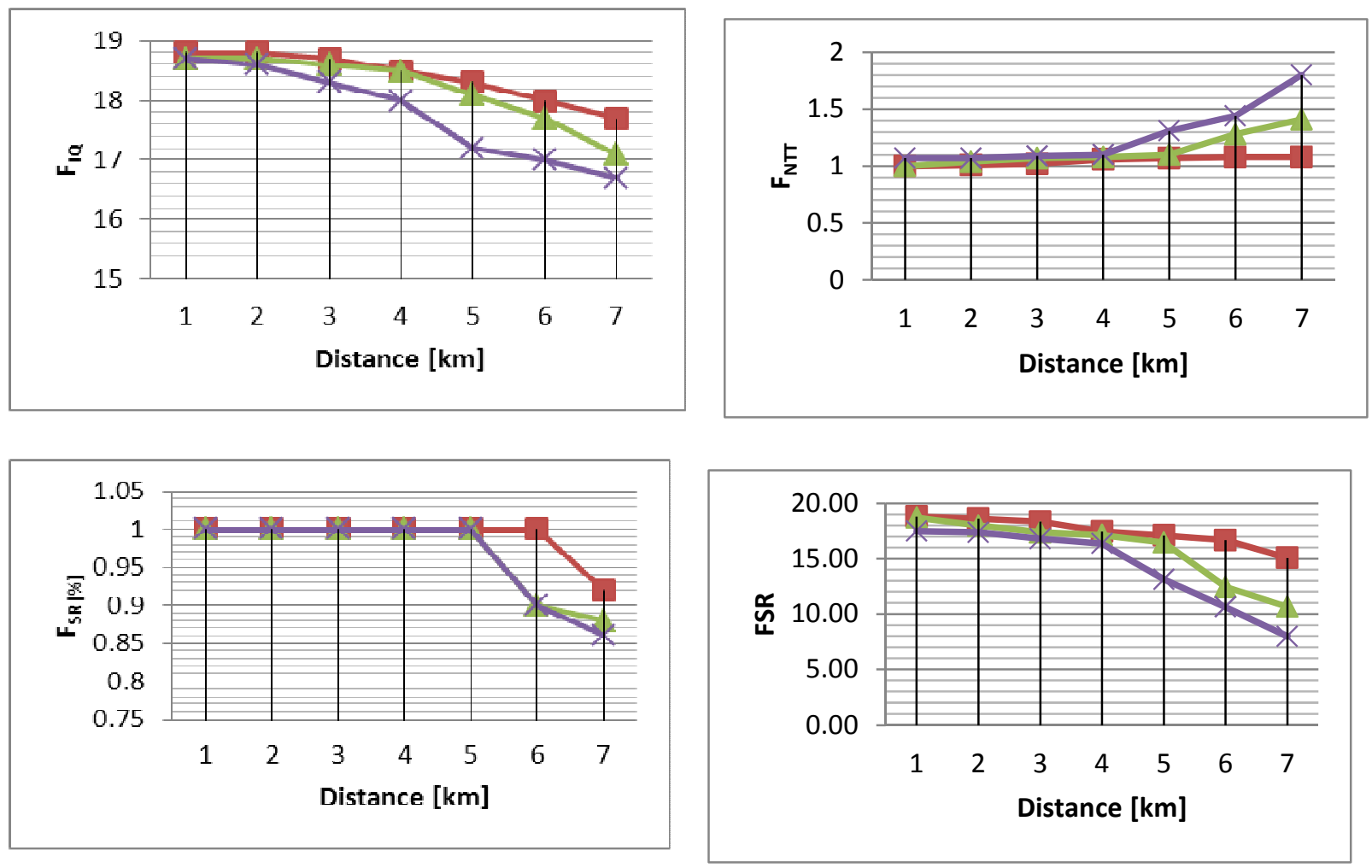

$\mathrm{Cap} / 15 \% \multimap \mathrm{Cap} / 60 \% \multimap \mathrm{Cap} / 85 \%$

Figure6. Influence of distance and traffic volume on the $\mathrm{F}_{\mathrm{IQ}}, \mathrm{F}_{\mathrm{NTT}}, \mathrm{F}_{\mathrm{SR}}$ and $\mathrm{FSR}$ parameters in urban area. 


\section{CONCLUSION}

The experiments discussed in this paper gave a clear indication that the facsimile transmission using GSM fixed wireless terminal is dependent on the terrain, the distance of the fixed wireless terminal from the base station and the traffic conditions [9]. The FSR parameter was used for measuring the quality of the facsimile transmission and it gave detailed information about the image quality under varying parameters. The FSR values indicate the margins of the analyzed GSM network's parameters that can be used as recommendations for the network designers to implement GSM networks through which facsimile service can also be offered.

\section{ACKNOWLEDGMENTS}

Our thanks to Mr. Sujith Kumar who have collaborated to the initiatives described in this paper.

\section{REFERENCES}

[1] ITU-T Rec. T.4, Standardization of Group 3 facsimile terminals for document transmission, July 2003.

[2] K. Toyoda, H. Ohno, J. Murai, D. Wing, A simple mode of facsimile using internet mail, IETF RFC 2305, March 1998

[3] Adegoke, A.S., I.T. Babalola, and W.A. Balogun, Performance Evaluation of GSM Mobile System in Nigeria, Pacific Journal of Science and Technology, Vol. 9, No.2, pp 436-441, November 2008.

[4] Tomaz Aljaz, Boyan Imperl, and Urban Mrak, The packet-based networks performance requirements for real-time facsimile transmission, Computer Communications, Vol. 30, No. 6, pp 1289-1300, March 2007.

[5] C.K. Yeo, S.C. Hui, I.Y. Soon and B.S. Lee, An adaptive protocol for real-time fax communications over internet, Computer Communications, Vol. 24, pp 1134-1146, October 2000.

[6] Ketan J. Sarvakar, Kiran Amin, Rajendra Patel, Menka Patel, Komal Maheta and Bhavesh Patel, Utilization of SIP contact header for reducing the load on proxy servers in FoIP application, in: First International Conference on Computational Intelligence, Communication Systems and Networks, July 2009.

[7] Umang Choudhary, Edward Perl and Deepinder Sidhu, Using T.38 and SIP for real-time fax transmission over IP networks, in: LCN 2001 Proceedings of the 26th Annual IEEE Conference on Local Computer Networks, November 2001.

[8] Zeeshan Irshad, Syed Junaid Nawaz and Dr. Sajjad Mohsin, A real-time system for multicast fax using IP network, in: CIT 2006 Proceedings of The Sixth IEEE International Conference on Computer and Information Technology, September 2006

[9] Kaveh Pahlavan, Prashant Krishnamurthy, Principles of Wireless Networks: A unified approach, PHI, 2010.

[10] ITU-T Rec. Q.700, Introduction to CCITT Signaling System No.7, March 1993

[11] Yi-Bang Lin, Imrich Chlamtac, Wireless and Mobile Network Architectures, Wiley, 2009.

[12] ITU-T Rec. V.32, A family of 2-wire duplex modems operating a data signaling rates of upto $9600 \mathrm{bit} / \mathrm{s}$ for use on the general switched telephone network and on leased telephone-type circuits, March 1993.

[13] ITU-T Rec. V.27 ter, 4800/2400 bits per second modem standardized for use in the general switched telephone network, November 1988

[14] http://www.tra.gov.om accessed on 11/08/2011

[15] ITU-T Rec. T.24, Standardized digitized image set, June 1998.

[16] Bilal Haider, M. Zafrullah, and M.K. Islam, Radio frequency optimization and QoS evaluation in operational GSM network, in: WCECS 2009 World Congress on Engineering and Computer Science, October 2009.

[17] Kuboye Bamidele Moses, Optimization models for minimizing congestion in Global System for Mobile Communications (GSM) in Nigeriation in operational GSM network, Journal Media and Communication Studies, Vol. 2, No.5, pp122-126, May 2010. 